\title{
Real-Time Power Management of Integrated Power Systems in All Electric Ships Leveraging Multi Time Scale Property
}

\author{
Gayathri Seenumani, Jing Sun, and Huei Peng
}

\begin{abstract}
All-electric ships (AES), enabled by integrated power systems (IPS), have been pursued for both commercial and military applications to meet the increasing ship-board power demand and environmental sustainability initiatives. They necessitate real-time power management (PM) for dynamic reconfiguration to support system critical operations in the event of dynamic load change or IPS component failures. The nonlinear, large scale trajectory optimization problem associated with IPS, along with the non-analytical nature of IPS model, makes many existing methods inadequate in meeting the real-time requirements. In this paper, we develop a methodology that exploits time scale separation, a characteristic associated with IPS dynamics, to achieve real-time optimization. In parallel, a dynamic model of the IPS with gas turbine and fuel cell as power plants is developed that captures the relevant dynamics but is simple enough for real-time optimization. The tradeoffs between the computational efficiency and optimization accuracy are analyzed. The optimization results for IPS PM on a real-time simulator are reported, which illustrate the real-time feasibility of the proposed optimization strategy.
\end{abstract}

Index Terms-All-electric ships (AES), integrated power systems (IPS), optimal power management, multitime scale property, realtime large scale optimization.

\section{INTRODUCTION}

$\mathbf{T}$ HE increasing power demand along with the mounting pressure on energy conservation and environment protection has driven the initiative to pursue all-electric ships (AES) [1] and have been pursued for both commercial and military applications. The integrated power systems (IPS) [2], [3] provides a common electrical platform to combine propulsion and ship service power system and hence enables the AES development. Such an architecture facilitates the integration of heterogenous power sources in order to achieve cleaner and more efficient power generation. An IPS, whose schematic is shown in Fig. 1, typically comprises of the following modules: power generation module (PGM), energy storage module (ESM), power conversion module (PCM), electric propulsion module (EPM), and ship service loads. For the IPS considered in this work, we consider gas turbine/generator and fuel cell as the power sources given their complementary efficiency and dynamic characteristics [4]. The ship service loads are further categorized into vital

Manuscript received April 17, 2010; revised November 21, 2010; accepted December 08, 2010. Manuscript received in final form January 17, 2011. Date of publication February 24, 2011; date of current version December 14, 2011. Recommended by Associate Editor L. Dessaint. This work was supported by ONR under the Award N00014-08-1-0611.

G. Seenumani and H. Peng are with the Department of Mechanical Engineering, The University of Michigan, Ann Arbor, MI 48109 USA (e-mail: gseenuma@umich.edu; hpeng@umich.edu).

J. Sun is with the Department of Naval Architecture and Marine Engineering, The University of Michigan, Ann Arbor, MI 48109 USA (e-mail: jingsun@umich.edu).

Digital Object Identifier 10.1109/TCST.2011.2107909 (such as communications, life support operations) and nonvital loads (such as space heating, entertainment).

In order to leverage the complementary characteristics of the power sources, coordination between them is essential. However, the interconnected nature of the IPS presents challenges in terms of managing individual components and their interactions. In this paper, we consider the optimal power management (PM) of the IPS targeting military applications. The control objective is to achieve optimal power split between the gas turbine and fuel cell in terms of energy efficiency and power tracking while ensuring component safety. The power management for the AES has to deal with the following problem characteristics:

- long time horizons associated with the transient and steady state missions;

- multiple power sources with complementary efficiency and time response characteristics;

- difficulty in obtained closed form representation of IPS dynamics, in addition to the nonlinear nature;

- requirements to enforce of component safety constraints. These features imply that the associated power management has to solve a large scale, nonlinear and constrained optimization problem. In addition, the On-demand nature of the problem for the military applications makes real-time control a key requirement, thereby making the optimal PM challenging.

The problem under consideration has been treated as a trajectory optimization problem for commercial hybrid land vehicles where many different optimization based methods have been explored (see a detailed survey in [5]). These methods include dynamic programming (DP) [6], stochastic dynamic programming (SDP) [7], and equivalent consumption minimization strategy (ECMS) [8]. While the "curse of dimensionality" makes the DP unsuitable for real-time execution, the lack of knowledge of a transition probability function makes the SDP and ECMS inapplicable for the control of IPS considered here. Sequential quadratic programming (SQP) [9] is the most well known gradient based method and has found wide applications in model predictive control [10]. While much work has been done in improving the efficiency of SQP [11] for large dimensional optimization problem, the applicability is mainly restricted to systems where second order derivatives can be computed analytically.

In this paper, we propose an optimization methodology based on the sensitivity function method [12], [13] for solving the power management of IPS in real-time. The main idea used to achieve real-time computational efficiency is to leverage the multitime scale property of the IPS and solve a two-level simplified optimization problem, where the control solutions at each level can be made available in real-time. Even though these solutions are suboptimal, it is shown that the reduction in computational delay is outweighs the effects of optimization accuracy 


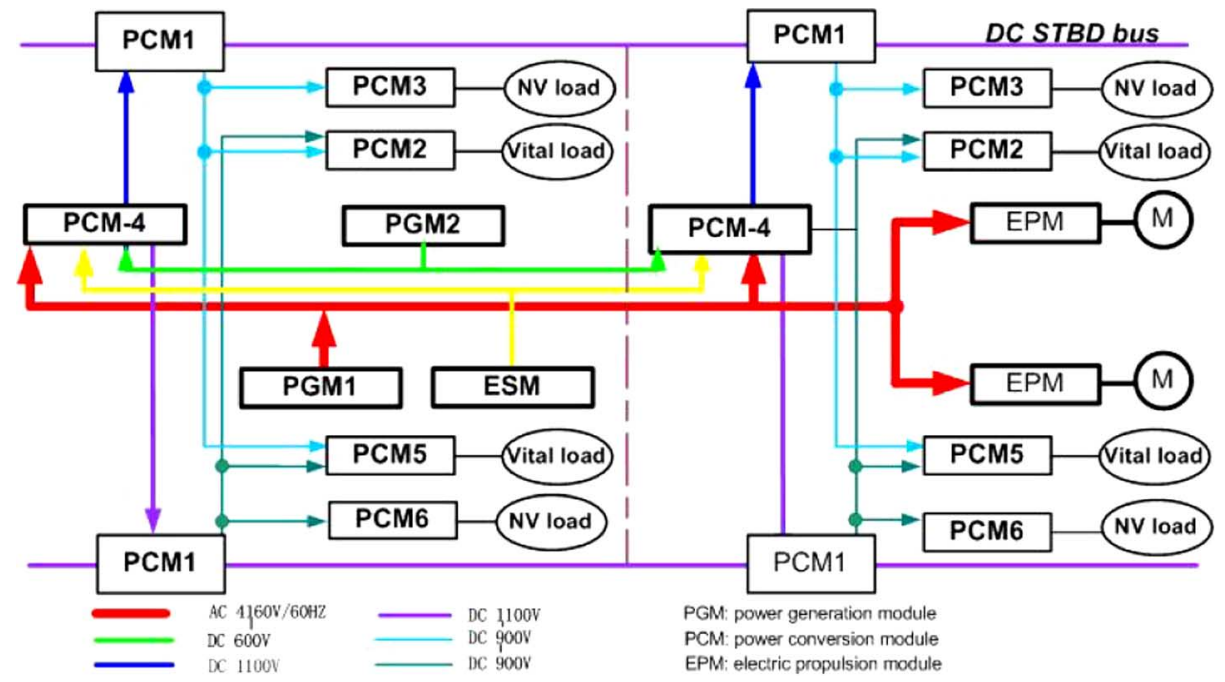

Fig. 1. Schematic of the IPS [3].

as illustrated in the later sections. First, we solve the optimization problem on the time scale of the slowest dynamics (fuel cell) and then seek transient corrections on the time scale involving the faster system dynamics. The key contributions are the realtime computational efficiency (namely, the control algorithm can be implemented at the required sampling rate without computational delay) of the proposed controller and the experimental validation results.

This paper is organized as follows. In Section II, the PM problem is formulated and the two time scale optimization algorithm is proposed. The dynamic model of the IPS is summarized in Section III. A case study of the IPS PM is developed in Section IV and the online optimization results with and without leveraging the multi time scale property are presented to illustrate the performance improvement of the proposed approach due to the timeliness of the optimization solutions. Section V concludes this paper with a discussion and summary of the main results.

\section{TRAJECTORY OPTIMIZATION METHODOLOGY FOR IPS POWER MANAGEMENT}

The optimization problem associated with the IPS power management is formulated and the two time scale methodology is proposed in this section. We consider a generic optimization for the problem formulation in order to capture the different requirements in the PM such as fast load-following, maximizing fuel economy and voltage regulation. We explore the sensitivity function method (SFM) [13], an iterative procedure, for solving constrained optimization problems associated with IPS power management. This method is chosen for the following two reasons: 1) it provides a mechanism to leverage the multi-time scale property of the IPS, for computational effort reduction and 2) the second-order derivatives, which are difficult to obtain due to the non-analytical nature of the IPS model, need not be computed.

\section{A. Optimization Problem Formulation}

For the problem formulation, we only consider the dynamics of the power sources (millisecond to second time constants), since they are much slower than those of power converters (microsecond time constants) and electric propulsion modules (millisecond time constants). Note that even among the power sources, the multi-time scale behavior can be observed, where the faster subsystem (e.g., gas turbine) is less efficient than the slower one (e.g., fuel cell). Therefore, in this work, we assume that there are two generic power plants, the fast and the slow ones and treat them as physically separated entities. We first describe the power plants dynamics and constraints before formulating the PM optimization problem.

Let $x_{1,2}, u_{1,2}$, denote the states and control inputs of the fast and slow sources. Let $f_{1}\left(x_{1}, u_{1}\right), f_{2}\left(x_{2}, u_{2}\right)$ denote the fast and slow power source dynamics. Then, the nonlinear IPS is described by

$$
x_{k+1}=f\left(x_{k}, u_{k}\right)
$$

where $x_{k}=\left[x_{1, k}^{T} x_{2, k}^{T}\right]^{T}\left(x \in \Re^{n}\right), u_{k}=\left[u_{1, k}^{T} u_{2, k}^{T}\right]^{T}(u \in$ $\left.\Re^{m}\right)$ and $f=\left[f_{1}\left(x_{1, k}, u_{1, k}\right)^{T} f_{2}\left(x_{2, k}, u_{2, k}\right)^{T}\right]^{T}$

We consider the following two types of constraints that the control algorithm has to enforce.

1) Component constraints denoted by $\Phi_{1}\left(x_{1, k}, u_{1, k}\right)$ and $\Phi_{2}\left(x_{2, k}, u_{2, k}\right)$ for the fast and slow subsystems. This includes the physical limits and the input saturation limits of the power sources.

2) Power generation constraints $\left(\Phi_{G}\left(x_{k}, u_{k}\right)\right)$ to ensure that the generation exceeds certain threshold.

The power management objectives are captured using a generic cost function $(J)$ which is given by

$$
J\left(x_{0}, u_{[0, N-1]}\right)=h\left(x_{N}\right)+\sum_{k=0}^{N-1} g\left(x_{k}, u_{k}\right)
$$

where $h\left(x_{N}\right) \geq 0$ is the terminal cost function, $g\left(x_{k}, u_{k}\right) \geq 0$ is the instantaneous cost function, $N$ is the time window over which the cost will be evaluated, $x_{k}, u_{k}$ are the instantaneous values of the states and controls at time $k$, respectively. We also use the notation $u_{[0, N-1]}=\left[u_{0}, u_{1}, \ldots, u_{N-1}\right]$ to denote the control sequence over the window. 
The optimization problem $\mathcal{P}_{N}$ is formulated as to find $u_{[0, N-1]}^{*}$, where

$$
\mathcal{P}_{N}: u_{[0, N-1]}^{*}=\arg \left\{\min _{u_{[0, N-1]}} J\left(x_{0}, u_{[0, N-1]}\right)\right\}
$$

subject to constraints (1) and

$$
\Phi\left(x_{k}, u_{k}\right) \leq 0
$$

where

$\Phi\left(x_{k}, u_{k}\right)=\left[\Phi_{1}\left(x_{1, k}, u_{1, k}\right)^{T} \Phi_{2}\left(x_{2, k}, u_{2, k}\right)^{T} \Phi_{G}\left(x_{k}, u_{k}\right)^{T}\right]^{T}$.

In the remainder of the section, we summarize the SFM, define the iterative procedure for solving optimization problem defined in $\mathcal{P}_{N}$, and quantify the computational effort associated with the method. We then propose a two-level optimization solution that exploits a specific structure of the SFM along with the multi time scale property to further reduce the computational effort.

Consider the performance index in (2), whose first order approximation can be expressed as

$J\left(u_{[0, N-1]}^{0}+\Delta u_{[0, N-1]}\right)=J\left(u_{[0, N-1]}^{0}\right)+\left.\sum_{i=0}^{N-1} \frac{\partial J}{\partial u_{i}}\right|_{u_{[0, N-1]}^{0}}$ where $u_{[0, N-1]}^{0}$ is any given control trajectory.

Let $J_{u_{i}}=\left.\left[(\partial J) /\left(\partial u^{1}\right) \ldots(\partial J) /\left(\partial u^{m}\right)\right]\right|_{\left(x_{i}, u_{i}\right)}, g_{u_{i}}=$ $\left.\left[(\partial g) /\left(\partial u^{1}\right) \ldots(\partial g) /\left(\partial u^{m}\right)\right]\right|_{\left(x_{i}, u_{i}\right)}$ and $g_{x_{i}}=$ $\left.\left[(\partial g) /\left(\partial x^{1}\right) \ldots(\partial g) /\left(\partial x^{n}\right)\right]\right|_{\left(x_{i}, u_{i}\right)}$, where $J_{u_{i}}, g_{u_{i}} \in \Re^{1 \times m}$ and $g_{x_{i}} \in \Re^{1 \times n}$. Then the sensitivity function defined as $(\partial J) /\left(\partial u_{[0, N-1]}\right)=\left[J_{u_{0}} J_{u_{1}} \ldots J_{u_{N-1}}\right]^{T}$ can be expressed as

$$
\frac{\partial J}{\partial u_{[0, N-1]}}=G_{u}+Z G_{x}
$$

where $Z \in \Re^{m N \times n(N+1)}, G_{x} \in \Re^{n(N+1)}$, and $G_{u} \in \Re^{m N}$ are defined as

$$
\begin{aligned}
G_{x} & =\left[\begin{array}{llll}
g_{x_{0}} & . . & g_{x_{N-1}} & g_{x_{N}}
\end{array}\right]^{T} \\
G_{u} & =\left[\begin{array}{lllll}
g_{u_{0}} & . . & g_{u_{N-1}}^{T}
\end{array}\right]^{T} \\
Z & =\left[\begin{array}{ccccc}
\mathbf{0} & B_{0}^{T} & \left(A_{1} B_{0}\right)^{T} & . . & \left(A_{N-1} \cdots A_{1} B_{0}\right)^{T} \\
\mathbf{0} & \mathbf{0} & B_{1}^{T} & . . & \left(A_{N-1} \cdots A_{2} B_{1}\right)^{T} \\
\ddot{.} & . . & . . & . . & . \ddot{0} \\
\mathbf{0} & \mathbf{0} & \mathbf{0} & . . & B_{N-1}^{T}
\end{array}\right]
\end{aligned}
$$

with $A_{i}=(\partial f) /\left(\partial x_{i}\right)$ and $B_{i}=(\partial f) /\left(\partial u_{i}\right)$ evaluated at $u_{[0, N-1]}^{0}, x_{[0, N]}^{0}$, where $x_{[0, N]}^{0}=\left[x_{0}^{0}, x_{1}^{0}, \ldots x_{N}^{0}\right]$ is the state trajectory sequence corresponding to the input $u_{[0, N-1]}^{0}$. The matrix $Z$ will be referred to as the state sensitivity matrix in the sense that $z_{i j}=\left(\left(\partial x_{i}\right) /\left(\partial u_{j}\right)\right)^{T}$.

Remark 2.1: Since the IPS model is nonlinear, in order to compute the $A_{i}$ and $B_{i}$ matrices, the dynamics need to be linearized. This is performed numerically given the non-closed form representation of the IPS model (e.g., compressor and turbine models are given as tables). For example, MATLAB offers a function linmod to determine the linearized dynamics around a given operating point.

The direction of search for the cost will either be in the steepest descent [13] or in the feasible direction [14] to deal with constraints. The feasible direction is defined as the steepest descent direction that does not violate any constraints. Let $\mathbf{K}^{a}$ denote the active set defined as

$$
\mathbf{K}^{a} \triangleq\left\{k \mid \Phi\left(x_{k}, u_{k}\right)=0, k \in[0: N-1]\right\} .
$$

For any given control sequence, if the active set $\mathbf{K}^{a}$ is empty, the search direction is $d^{*}=-\nabla J$. Otherwise, in the neighborhood of a given control sequence, the feasible descent direction $d^{*}$ will be the solution to a linear programming problem, defined as follows:

$$
\min _{d_{[0: N-1]}, l} l
$$

subject to constraints

$$
\begin{aligned}
\nabla J^{T} d-l & \leq 0, \\
\nabla \Phi_{a}^{T} d-l & \leq 0, \\
-1 & \leq\|d\|_{\infty} \leq 1
\end{aligned}
$$

Where minimizing $l$ leads to reducing the cost as well as the $u_{i}$ constraint violation in the direction $d$. Here $\nabla J, \nabla \Phi_{a}$ are the normalized gradient of the cost function and active constraint set respectively and are defined as follows:

$$
\begin{aligned}
\nabla J & \triangleq \frac{\partial J}{\partial u_{[0: N-1]}} / \max \left|\frac{\partial J}{\partial u_{[0: N-1]}}\right| \\
\nabla \Phi_{a} & \triangleq \frac{\partial \Phi\left(x_{k}, u_{k}\right)}{\partial u_{[0: N-1]}} / \max \left|\frac{\partial \Phi\left(x_{k}, u_{k}\right)}{\partial u_{[0: N-1]}}\right|, \quad \forall k \in \mathbf{K}^{\mathbf{a}}
\end{aligned}
$$

where $\max |y|$ for any vector $y \in R^{N}$ is defined as $\max \left(\left|y_{1}\right|,\left|y_{2}\right|, \ldots,\left|y_{N}\right|\right)$.

The control updates are then computed by performing a 1-D search over the step size $\delta$ as follows:

$$
\delta^{*}=\arg \left(\min _{\delta} J\left(u_{[0: N-1]}+\delta d^{*}\right), \text { s.t., } 0<\delta \leq \delta_{0}\right.
$$

where $\delta_{0}$ is chosen such that the updates are in the neighborhood of the control sequence $\left(\left\|\delta_{0} d_{k}^{*}\right\| \ll 1\right)$ and constraints (3) are satisfied.

Remark 2.2: The state sensitivity matrix $Z$ is upper triangular as the system is causal. In addition, for a stable system, the $Z$ matrix will be sparse and has a band diagonal structure, where the number of band diagonal elements is defined as the index $N_{B}$ such that $\forall i>N_{B},\left\|\prod_{k=1}^{i} A_{k}\right\| \approx 0$. The faster the dynamics, the more sparse the $Z$ matrix.

Remark 2.3: Even though the first order methods have been used, leveraging the band diagonal structure reduces the computations required to determine the sensitivity functions, hence the control updates. This will especially be useful in solving optimization problems with extended horizon.

We now propose an iterative procedure for trajectory optimization of systems:

\section{Algorithm I (Sensitivity function method):}

Given a feasible initial control trajectory $u_{[0, N-1]}$, tolerance $\sigma>0$ 
1: Simulate the dynamic model, determine the state trajectories and compute the performance index $J\left(u_{[0, N-1]}\right)$.

2: Compute the sensitivity matrices $Z, G_{x}, G_{u}$ and using (5), compute the sensitivity functions, $\left((\partial J) /\left(\partial u_{[0, N-1]}\right)\right)$, with the band diagonal structure of size $N_{B}$. (See Remark 2.2).

3: Determine the active set $\mathbf{K}^{a}$. If the active set is empty, set $d^{*}=-\nabla J$, otherwise compute $d^{*}$ by solving (8).

4: Compute optimal step size $\delta^{*}$ by solving (9) and calculate the optimal update vector as $\Delta u_{[0, N-1]}^{*}=\delta^{*} d^{*}$.

5: If $J\left(u_{[0, N-1]}\right)-J\left(u_{[0, N-1]}+\Delta u_{[0, N-1]}^{*}\right)<\sigma$, stop. Otherwise update $u_{[0, N-1]}=u_{[0, N-1]}+\Delta u_{[0, N-1]}^{*}$ and go to step (1).

In Algorithm I, we assume perfect state measurements are available for the power plants considered in the shipboard IPS, namely gas turbine and the fuel cell states, as is currently the case with most power generation units.

Proposition 2.1: For a given number of states $(n)$ and control inputs $(m)$, the computational effort $C_{S F}$ (flop count) required per iteration of Algorithm I when $N_{B}=N$ and $N \gg m, n$ is $\mathcal{O}\left(N^{2}\right)$.

The key ideas used in deriving Proposition 2.1 are given in Appendix A.

Corollary 2.1: Let $C_{B D}$ denote the computational effort (flop count) for sensitivity function generation when we leverage band diagonal structure, i.e., we assume a sparse structure for the $Z$ matrix and set upper triangle elements as zero, then, the ratio $\left(C_{B D}\right) /\left(C_{S F}\right)$ is of $\mathcal{O}\left(N_{B} / N\right)$.

Proof: See Appendix $B$.

Remark 2.4: One possible way of choosing the initial control sequence $u_{[0, N-1]}$ is using the steady state optimal control input, which is determined by solving $\mathcal{P}_{N}$ as a static optimization problem. However such a choice may not satisfy the constraints (4). Therefore, in this work, the initial control sequence is chosen either as the unfiltered or the filtered steady-state optimal control, where the latter is used when the constraints are violated.

Remark 2.5: The tolerance parameter $(\sigma)$ used in the stopping criteria for the Algorithm $I$ is typically a small parameter [14] and it influences the optimization accuracy as well as the computational effort. While the exact value depends on the cost function $J$, in this work this value is chosen such that $\sigma J\left(x_{0}, u_{[0: N-1]}^{0}\right) \leq 10^{-8}$.

\section{B. Proposed Two Time Scale Optimization}

Since the complexity of Algorithm I is $\mathcal{O}\left(N^{2}\right)$, we seek ways to simplify the dimensions of the optimization problem $\mathcal{P}_{N}$ as this would yield considerable savings in the computational effort. To do so, we exploit the multitime scale property and consider a two-level optimization approach, where the optimal solutions at each level is the initial control sequence for subsequent optimization. The potential benefits of such an approach is that the control solutions at each level can be made available much earlier than the optimal control solutions $\left(u_{[0, N-1]}^{*}\right)$ computed using Algorithm I. On the top-level, we simplify the optimization problem $\mathcal{P}_{N}$ on the time scale of the slower power source dynamics. Then, to account for under-sampling the fast dynamics on the slow time scale, we seek transient corrections on the time scale of the faster power source. It must be noted that the proposed two time scale optimization in this paper deals with multitime scale and decoupled subsystems.

Let $\tau_{f}, T_{f}$ and $\tau_{s}, T_{s}$ denote the time constant and the sampling time for the fast and slow subsystems, respectively. If the total time interval is $t_{f}$ and $\rho=\left(T_{s}\right) /\left(T_{f}\right)$ is an integer, ${ }^{1}$ we now consider the following two optimization problems.

1) Level 1 Optimization $\left(\mathcal{P}_{N_{s}}^{s}\right)$ : At this level, we solve the problem $\mathcal{P}_{N}$ on the time scale determined by the slower power source. The problem is formulated to find the optimal control sequence $u_{\left[0: N_{s}-1\right]}^{s, *}$ on the slow time scale given by

$$
\mathcal{P}_{N_{s}}^{s}: u_{\left[0: N_{s}-1\right]}^{s, *}=\arg \left\{\min _{u_{\left[0, N_{s}-1\right]}^{s}} J\left(x_{0}, u_{\left[0: N_{s}-1\right]}^{s}\right)\right\}
$$

subject to constraints

$$
\begin{aligned}
& x_{k_{s}+1}=f\left(x_{k_{s}}, u_{k_{s}}\right) \\
& \Phi\left(x_{k_{s}}, u_{k_{s}}\right) \leq 0
\end{aligned}
$$

where $k_{s}$ is the discrete time index on the slower time scale and $N_{s}=\left(t_{f}\right) /\left(T_{s}\right)$ is the size of the time window on the slower time scale. Then, level 1 solution is the control sequence $u_{1,[0: N-1]}, u_{2,[0: N-1]}$ given by

$$
\begin{aligned}
& u_{1, k}=u_{1, k_{s}}^{*}, \quad \forall \rho k_{s} \leq k \leq \rho\left(k_{s}+1\right) \\
& u_{2, k}=u_{2, k_{s}}^{*} .
\end{aligned}
$$

Remark 2.6: Under the assumption that the dynamics of the subsystem 1 are much faster than those of subsystem 2, we have $N_{s} \ll N$ and make the following observations.

- In solving (10), the dynamics of the faster subsystem are under-sampled at the rate designed for the slower time scale. Hence the level 1 solution $\left(u_{k}\right)$, are treated as suboptimal and expect that the true optimal solution $u_{k}^{*} \approx u_{k}$ for all instants except around the transients.

- Since the SFM algorithm is $\mathcal{O}\left(N^{2}\right)$, the optimization problem $\mathcal{P}_{N_{s}}^{s}$ defined by (10)-(12), is simpler than (3) and (4). The effort can be reduced further if the band diagonal structure is used.

2) Level 2 Optimization $\left(\mathcal{P}_{N}^{f}\right)$ : This problem is formulated on the fast time scale $\left(N=\left(t_{f}\right) /\left(T_{f}\right)\right.$, to account for undersampling the faster dynamics on Level 1 and computes the corrections $u_{1,[0: N-1]}^{f, *}=\arg \left(J_{f}\right)$, where $J_{f}$ is computed as

$$
\mathcal{P}_{N}^{f}: J_{f}=\min _{u_{1,[0, N-1]}^{f}} J\left(x_{0}, u_{1,[0: N-1]}+u_{1,[0: N-1]}^{f}, u_{2,[0: N-1]}\right)
$$

subject to constraints

$$
\begin{aligned}
& x_{1, k+1}=f_{1}\left(x_{1, k}, u_{1, k}+u_{1, k}^{f}\right) \\
& \Phi_{1}\left(x_{1, k}, u_{1, k}+u_{1, k}^{f}\right) \leq 0
\end{aligned}
$$

${ }^{1} T_{s}, T_{f}$ can be chosen such that $\rho$ is an integer and satisfy the Nyquist criteria, i.e., $T_{f} \leq\left(\tau_{f}\right) /(2), T_{s} \leq\left(\tau_{s}\right) /(2)$. 


$$
\Phi_{G}\left(x_{1, k}, u_{1, k}+u_{1, k}^{f}\right) \leq 0
$$

where $x_{1,[0: N]}$ are the states associated with the control inputs $\left(u_{1,[0: N-1]}+u_{1,[0: N-1]}^{f}\right)$ to the fast subsystems, with $u_{1,[0: N-1]}$ computed using (13). Note that based on Remark 2.6, the initial control sequence for the level 2 optimization is chosen as $\mathbf{0}_{1 \times N-1}$.

Remark 2.7: If SFM method is used to solve the optimization defined in $\mathcal{P}_{N}^{f}$, since only the fast dynamics are considered the sparsity of the $Z$ matrix will be high, thereby making $\mathcal{P}_{N}^{f}$ much simpler to solve than $\mathcal{P}_{N}$ (See Remark 2.2).

\section{Algorithm II:}

Given a feasible initial control trajectory $u_{[0, N-1]}^{0}$ (See Remark 2.4), we propose the following algorithm to solve the optimization problem $\mathcal{P}_{N}$ defined by (3):

1) At $\mathrm{k}=0$, determine $u_{[0, N-1]}^{s, *}$ by solving the optimization problem $\mathcal{P}_{N_{s}}^{s}$ using SFM and compute the sub-optimal control trajectories $u_{[0, N-1]}$ using (13), (14).

2) Given $u_{[0, N-1]}$ and using $0_{1 \times N-1}$ as the initial control sequence, compute $u_{[0: N-1]}^{f, *}$ by solving the optimization problem $\mathcal{P}_{N}^{f}$ using SFM.

3) If $\mathcal{P}_{N}^{f}$ can be solved in real time: Compute $u_{1,[0, N-1]}+u_{[0, N-1]}^{f, *}$ and $u_{2,[0, N-1]}$ as the sub-optimal control inputs to the power plants.

4) Else: Compute $u_{1,[0, N-1]}$ and $u_{2,[0, N-1]}$ as the sub-optimal as the sub-optimal control inputs to the power plants.

Computational Efficiency Versus Optimization Accuracy: Consider the following three possible solutions (there are more than three solutions, we only consider three) to the IPS power management problem, namely, as follows.

FS Full Scale (FS) control sequence obtained by solving the optimization problem defined in $\mathcal{P}_{N}($ Algorithm I).

L1 Level 1 control sequence obtained by solving the optimization problem defined in $\mathcal{P}_{N_{s}}^{s}$, i.e., step 1 of Algorithm II.

L2 Level 2 control sequence obtained by solving the optimization problem defined in $\mathcal{P}_{N}^{f}$, i.e., steps 1 and 2 of Algorithm II.

While the FS solutions have the best optimization accuracy, followed by the $L 2, L 1$ and the steady-state solutions, this order is reversed with respect to the computational effort required to solve these problems. However, the ability to solve the optimization problem in real-time without computational delay is another key factor in deciding the performance (i.e., optimization accuracy) associated with these approaches. Therefore the most suitable algorithm is the one that can achieve a good tradeoff between the real-time computational efficiency and optimization accuracy. In light of this, we attempt to quantify the reduction in computational effort associated with computing the solutions using L1 and L2 as compared to the full scale (FS) approach. We
TABLE I

GT MODELING NOMENCLATURE

\begin{tabular}{|c|c|}
\hline Variable & Description (Unit) \\
\hline$c_{p, a i r}, c_{p, f}, c_{p, g a s}$ & $\begin{array}{c}\text { Specific heat at constant pressure } \\
\text { of air, fuel and burnt gas }(\mathrm{kJ} / \mathrm{kg} \mathrm{K}) . \\
\text { Constant volume specific heat } \\
\text { for burner material }(\mathrm{kJ} / \mathrm{kg} \mathrm{K}) .\end{array}$ \\
\hline$J_{I}$ & Shaft inertia $\left(\mathrm{Kgm}^{2}\right)$ \\
\hline$m_{b}$ & Mass inside burner $(\mathrm{Kg})$, \\
$M_{b, i n}$ & Molecular mass of air-fuel \\
& mixture inside burner $(\mathrm{Kg} / \mathrm{mole})$ \\
\hline$p_{a m b}, p_{b}$ & Ambient and combustor pressure (Pa) \\
\hline$P_{c}, P_{t}$ & Compressor and Turbine Power $(\mathrm{KW})$ \\
\hline$Q_{L H V}$ & $J P_{5}$ lower heating value $(\mathrm{kJ} / \mathrm{kg})$ \\
\hline$R$ & Universal Gas constant (kJ/K mol), \\
\hline$T_{a m b}, T_{r e f}$, & Ambient,reference, compressor \& \\
$T_{c, o u t}, T_{t, o u t}, T_{b}$ & turbine outlet, combustor temperature $(\mathrm{K})$ \\
\hline$V_{b}$ & Burner Volume $\left(\mathrm{m}^{3}\right)$ \\
\hline$W_{f}, W_{t}, W_{c}$ & Fuel flow, Compressor, Turbine air flow (kg/s) \\
\hline$\tau_{d e m}, \omega_{t c}$ & Load Torque (N-m), Shaft rotational speed (rad/s) \\
\hline$\gamma$ & Specific heat ratio \\
\hline$\eta_{m}$ & Turbine mechanical \\
\hline$\eta_{i s, c}, \eta_{i s, t}$ & Compressor, turbine isentropic efficiency \\
\hline
\end{tabular}

rely on the assumption that sufficient difference in the dynamics of fast and slow subsystems exist, the optimization accuracy of the $L 1$ or $L 2$ can be comparable to the $F S$ solutions, where the benefits of the improved computational efficiency can achieve better real-time performance. We now provide estimates of the computational effort reduction in the following proposition.

Proposition 2.2: Let $C_{\mathrm{FS}}, C_{L 1}, C_{L 2}$ denote the flop count per iteration using $F S, L 1$, and $L 2$ optimization respectively and $\varepsilon \triangleq\left(N_{s}\right) /(N)$ capture the multi time scale property. Under the assumption $\varepsilon \ll 1$, we have (a) $\left(C_{L 1}\right) /\left(C_{\mathrm{FS}}\right)=\mathcal{O}\left(\varepsilon^{2}\right)$ and (b) $\left(C_{L 2}\right) /\left(C_{\mathrm{FS}}\right)=\mathcal{O}(\varepsilon)$.

Proof: See Appendix $C$

The effectiveness of the proposed algorithm and the validity of the estimates in effort reduction will be demonstrated on the IPS optimization in Section IV.

\section{OPTIMIZATION-ORIENTED IPS MODEL}

In this section, we develop an control-oriented dynamic model of the IPS in order to apply the algorithm proposed in Section II. The IPS model consists of the component models, namely, gas turbine and fuel cell whose dynamics are briefly summarized below. More detailed model can be found in [15].

\section{A. Gas Turbine}

The gas turbine model captures the dynamic characteristics of compressor, turbine, combustor and the coupling between turbine and compressor. The combustor mass and temperature dynamics along with turbocharger rotational dynamics are considered, while the compressor and turbine air mass flows and efficiencies and obtained by curve fitting the performance maps. The variables and parameters used in GT model are defined in Table I.

1) Compressor, Turbine: The compressor power is determined using first law of thermodynamics and is given by

$$
P_{c}=W_{c} c_{p}\left(T_{c, \text { out }}-T_{\mathrm{amb}}\right)
$$

where the compressor exit temperature $T_{c, \text { out }}$ is given as 
TABLE II

FC MODELING NOMENCLATURE

\begin{tabular}{|c|c|}
\hline Variable & Description \\
\hline$c_{p, F R}$ & $\begin{array}{c}\text { Constant ratio specific heat of FR } \\
\text { material }(\mathrm{kJ} / \mathrm{kg} \mathrm{K})\end{array}$ \\
\hline$E$ & Open circuit Fuel cell voltage \\
\hline$h_{\text {in }}, h_{\text {out }}$ & $\begin{array}{l}\text { Specific enthalpy }(\mathrm{J} / \mathrm{kg}) \text { of inlet } \\
\text { and outlet FR flows }\end{array}$ \\
\hline$m_{F R}$, & Mass inside the reformer unit $(\mathrm{kg})$ \\
\hline$M_{a n}, M_{H_{2}}$ & $\begin{array}{c}\text { Molecular mass of anode material } \\
\text { and hydrogen }(\mathrm{kg} / \mathrm{mol})\end{array}$ \\
\hline$n_{c}$ & Number of fuel cells in the stack \\
\hline$N_{\text {in }}, N_{\text {out }}$ & Molar flow rates in and out of the FR $(\mathrm{mol} / \mathrm{s})$ \\
\hline$p_{a n}, p_{H_{2}, a n}$ & Anode total and partial pressure $(\mathrm{Pa})$ \\
\hline$R$ & Universal gas constant $(\mathrm{J} / \mathrm{K} \mathrm{mol})$ \\
\hline$T_{F R}, T_{a n}$ & FR and anode temperature (K) \\
\hline$v_{\text {ohm }}, v_{\text {act }}, v_{\text {conc }}$ & $\begin{array}{c}\text { Ohmic, activation and } \\
\text { concentration loss respectively (volt) }\end{array}$ \\
\hline$V_{a n}$ & Anode volume $\left(\mathrm{m}^{3}\right)$ \\
\hline $\begin{array}{c}W_{F R, \text { in }} \\
W_{F R, H_{2}}, W_{F R, \text { out }}\end{array}$ & $\begin{array}{l}\text { Total flow into the FR }(\mathrm{kg} / \mathrm{s}) \\
\text { Hydrogen and total flow out of FR }(\mathrm{kg} / \mathrm{s})\end{array}$ \\
\hline$W_{H_{2}, \text { react }}$ & Reacted Hydrogen inside anode $(\mathrm{kg} / \mathrm{s})$ \\
\hline$W_{H_{2}, a}$ & Hydrogen and total anode exit flow $(\mathrm{kg} / \mathrm{s})$ \\
\hline
\end{tabular}

\section{move text here}

$$
T_{c, \text { out }}=T_{\mathrm{amb}}\left[1+\frac{1}{\eta_{i s, c}}\left(\left(\frac{p_{b}}{p_{\mathrm{amb}}}\right)^{\frac{\gamma-1}{\gamma}}-1\right)\right] .
$$

Similarly the turbine power is given by

$$
P_{t}=W_{t} c_{p}\left(T_{b}-T_{t, \text { out }}\right)
$$

where

$$
T_{t, \text { out }}=T_{b}\left[1-\eta_{\text {is }, t}\left(1-\left(\frac{p_{\mathrm{amb}}}{p_{b}}\right)^{\frac{\gamma-1}{\gamma}}\right)\right] .
$$

2) Combustor: The burner mass is determined using the mass balance and is given by

$$
\dot{m}_{b}=W_{f}+W_{c}-W_{t}
$$

and the temperature is determined using energy balance as

$$
\begin{aligned}
\dot{T}_{b}= & \frac{1}{m_{b} c_{v, b}}\left(\left(W_{c} c_{p, \text { air }}+W_{f} c_{p, f}\right)\left(T_{c, \text { out }}-T_{\text {ref }}\right)\right. \\
& \left.+W_{f} Q_{\mathrm{LHV}}-W_{t} c_{p, \text { gas }}\left(T_{b}-T_{\text {ref }}\right)\right) \\
& \left.-c_{v, b}\left(T_{b}-T_{\text {ref }}\right) \dot{m}_{b}\right) .
\end{aligned}
$$

The burner pressure $p_{b}$ is derived using ideal gas law $p_{b}=$ $\left(m_{b}\right) /\left(M_{b, \text { in }} V_{b}\right)\left(R T_{b}\right)$.

3) Turbocharger: The shaft speed is determined using the power balance on the shaft given by

$$
\dot{\omega}_{t c}=\frac{1}{J_{I}}\left(\frac{\eta_{m} P_{t}-P_{c}}{\omega_{t c}}-\tau_{\mathrm{dem}}\right) .
$$

\section{B. Fuel Cell and Reforming Unit}

We consider a polymer electrolyte membrane (PEM) fuel cell along with a fuel processing system (FPS). The FPS + FC system [16] consists of a hydro desulphurizer (HDS), heat exchanger (HEX), mixer (MIX), fuel reformer (FR) that converts the fuel flow to pure hydrogen, water gas shift reactor (WGS) for gas clean up and fuel cell anode dynamics. A detailed 10 state model of the FPS+FC system along with relevant assumptions has been developed in [16]. In this work, we summarize a three state reduced order model that captures the system dynamics as well as operating constraints such as fuel starvation.

1) Hydro Desulphurizer: The HDS is represented as a large volume and is simplified as a first order lag with a large time constant $\left(\tau_{\text {HDS }}=5 \mathrm{~s}\right)$ that reflects the slow dynamics of the linearized model given in [16], the other two slow dynamics being the FR temperature and the anode hydrogen partial pressure.

2) Fuel Reformer: The FR model is developed in [16] and is summarized here. The temperature dynamics using energy balance is given by

$$
\frac{d T_{\mathrm{FR}}}{d t}=\frac{1}{m_{\mathrm{FR}} c_{p, \mathrm{FR}}}\left[N_{\mathrm{in}} h_{\mathrm{in}}-N_{\text {out }} h_{\mathrm{out}}\right]
$$

where $N_{\text {in }}$ is the inlet flow (fuel and air flow) and $N_{\text {out }}$ is the outlet flow is the sum of the flows of the following species: $\mathrm{CH}_{4}$, $\mathrm{CO}, \mathrm{CO}_{2}, \mathrm{H}_{2}, \mathrm{H}_{2} \mathrm{O}, \mathrm{N}_{2}$.

3) Anode: The anode partial pressure dynamic using mass balance is given by

$$
\begin{aligned}
\frac{d p_{H_{2}, a n}}{d t} & =\frac{R T_{a n}}{M_{H_{2}} V_{a n}}\left(W_{\mathrm{FR}, H_{2}}-\chi_{H_{2}}^{a n} W_{a n}-W_{H_{2}, \text { react }}\right), \\
\chi_{H_{2}}^{a n} & =\left(\frac{M_{H_{2}}}{M_{a n}}\right)\left(\frac{p_{H_{2}, a n}}{p_{a n}}\right)
\end{aligned}
$$

where $W_{\mathrm{FR}, H_{2}}$ is the hydrogen flow from the reformer, $W_{a n}$, $W_{\mathrm{H}_{2} \text {,react }}$ are the anode outlet flow and reacted hydrogen as given in [16]. The air supply is assumed to be instantaneous and the cathode pressure follows the anode pressure. The readers are referred to [16] for more information on the stack voltage model and other details.

Remark 3.1: The control inputs to the HPS model are the fuel flow to the gas turbine and fuel cell along with the battery current. The total power produced by the HPS is the sum of the power generated by the sources and battery.

\section{IPS Efficiency and Time Response Characteristics}

For the IPS model, we consider an arrangement of eight fuel cell modules connected in series is considered to match the GT turn-off ratio. Table III summarizes the ranges of power output, fuel input, corresponding efficiencies and the spectrum of associated linearized models of the gas turbine and fuel cell. From Table III, it can be seen that FC is much more efficient than the GT. In order to understand the multitime scale property between GT and FC, given the nonlinearity of the IPS model, we consider two measures: 1) The spectral analysis of the linearized GT and FC models derived at different operating condition and 2) The settling time of normalized step response (from fuel to power). It can be seen that even though there is 10 times separation between the fast eigenvalues of the gas turbine and fuel cell respectively, there is an overlap in the spectrum. However, from Fig. 2, it can be seen that the settling time of gas turbine (1.5 s) is about 5 times faster than the fuel cell $(7 \mathrm{~s})$ which indicates separation in the scale of their time responses. It can be seen that 


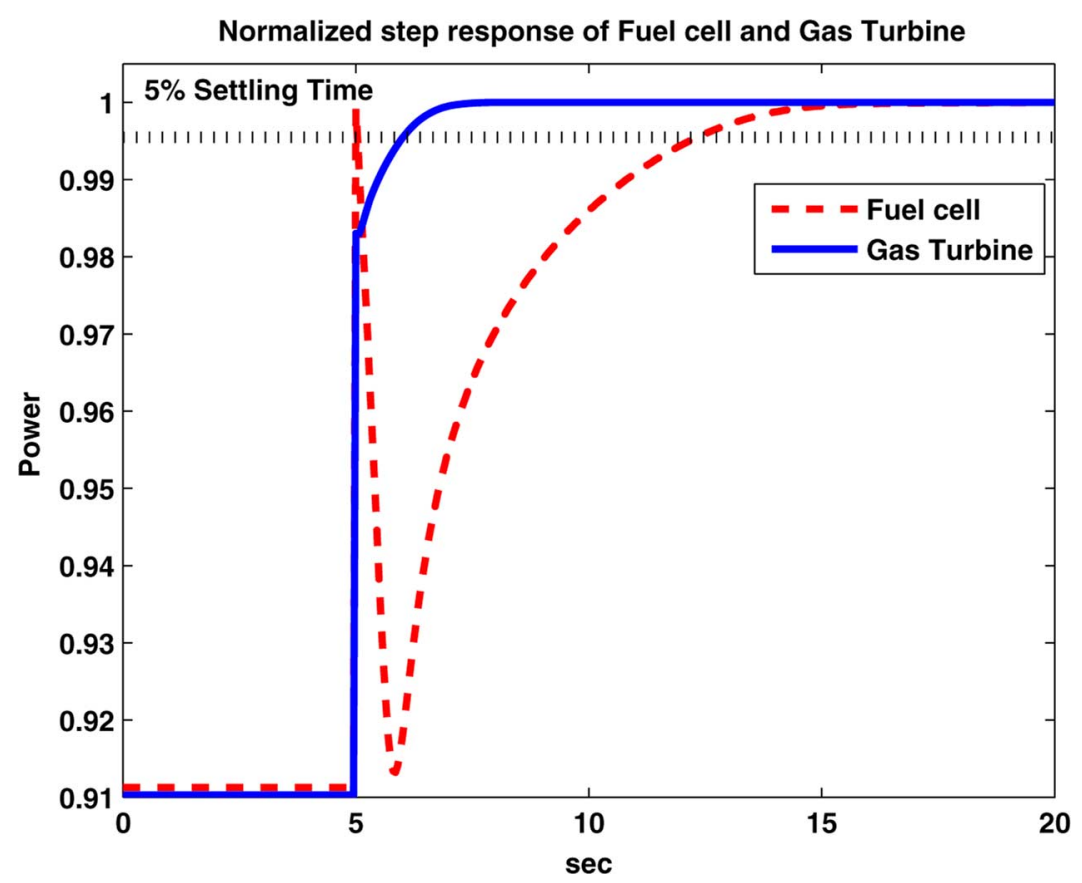

Fig. 2. Normalized step response of fuel cell and gas turbine.

TABLE III

FC, GT PRINCIPAL CHARACTERISTICS

\begin{tabular}{|c|c|c|}
\hline Variable & Fuel Cell & Gas Turbine \\
\hline Power & $484-1838 K W$ & $1187-4122 K W$ \\
\hline Fuel limits & $0.022-0.124 \mathrm{~kg} / \mathrm{s}$ & $0.137-0.345 \mathrm{~kg} / \mathrm{s}$ \\
\hline Corresponding Efficiency & $35-51 \%$ & $18.5-28.1 \%$ \\
\hline Corresponding Eigen-Values & $s_{1} \in[-2.2-0.5]$ & $s_{1} \in[-0.5-1]$ \\
& $s_{2} \in[-1.5-0.26]$ & $s_{2} \in[-9-24]$ \\
& $s_{3}=-0.2$ & $s_{3} \in[-12-24]$ \\
\hline
\end{tabular}

the fuel cell and gas turbine exhibits complementary characteristics in terms of system efficiency and transient response.

\section{CASE Study}

A case study is developed in order to solve the PM using the IPS model developed in the previous section. The objectives of the power management in this case study is to support the load demands as quickly and as efficiently as possible, where the loads are represented as time varying power demands. We consider a hypothetical pulse power profile (see Fig. 3, black solid line) associated with an aircraft or a weapon launch scenario. The terminal and the instantaneous cost function (see Table IV) captures the PM objectives, where the latter is the weighted sum of power tracking error and total fuel consumption. The constraints along with the other optimization parameters are given in Table IV. Here $u_{1, k}, u_{2, k}$ are the fueling rates to the gas turbine and fuel cell and $P_{1, k}, P_{2, k}$ are the associated power outputs.

The main purpose of the case study is to illustrate the benefits of the multitime scale approach by comparing the FS and $L 1$ solutions in terms of the following aspects.

- Comparable optimization accuracy of the $L 1$ with respect to the FS approach, when the optimization problems were solved offline, which is due to the complementary dynamic response characteristics of the power sources.
- Real-time computational efficiency of the $L 1$ optimization as compared to the FS one, a direct consequence of which is an improved real-time power tracking performance of the $L 1$ optimization.

The implementation of the controller was done both offline and in real-time where we used a Pentium processor for the offline and a dual core OpalRT realtime target for online optimization and the corresponding results are presented in Table V. The first point to be noted is that the optimization accuracy of the $L 1$ solutions is only about $3 \%$ lesser than the full-scale solutions, when both the optimization problems were solved offline. However it can seen (Column 3) that the real-time accuracy of the $L 1$ solution (4.6) is much better than the $F S$ one (30.2) due to the computational efficiency. While it takes $0.045 \mathrm{~s}\left(<T_{f}\right)$ to get the control solutions of the $L 1$ optimization, the corresponding FS problem takes $1.6 \mathrm{~s}\left(\gg T_{f}\right)$ to be solved, during which the static optimal control input is applied to the power sources. This leads to a performance deterioration of the FS solution in terms of power tracking. The actual computational reduction from FS to $\mathrm{L} 1$ optimization is 32 which is $\mathcal{O}\left(\varepsilon^{2}\right)\left(\varepsilon^{2}=16\right)$ and is in line with the estimation given by Proposition. 2.2.

Fig. 3 shows the real power tracking and the associated control trajectories, namely the fueling rates for the gas turbine and fuel cell. The power output $P_{S S}$ associated with the steady state control is denoted by the green dashed line, while the power 

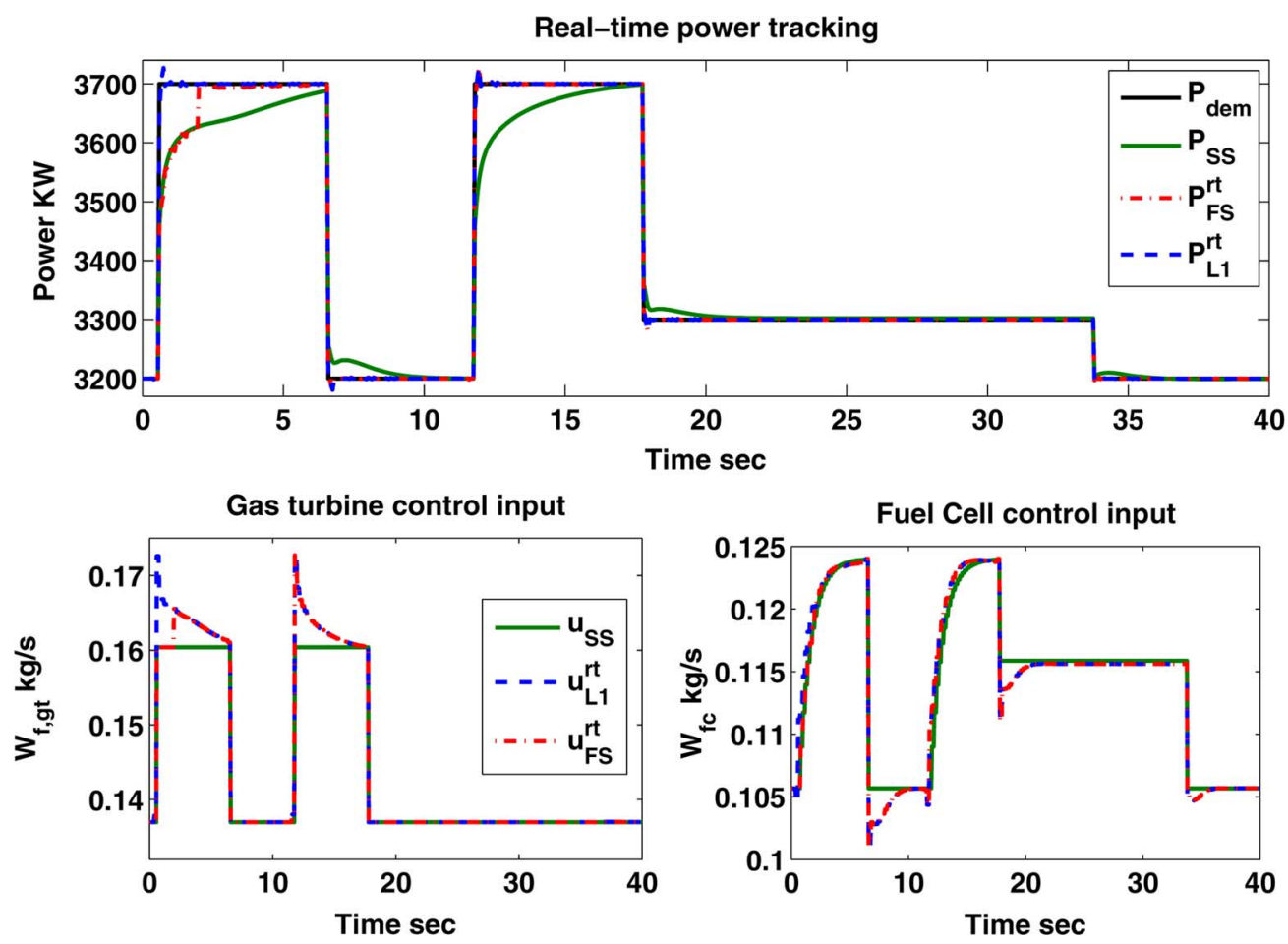

Fig. 3. Power and control trajectories: Demanded power $\left(P_{\text {dem }}\right)$, real-time power tracking with static feed-forward optimal control $u_{S S}\left(P_{S S}\right)$, Level 1 optimal control $u_{L 1}^{r t}\left(P_{L 1}^{r t}\right)$ and true optimal control $u_{\mathrm{FS}}^{r t}$ using full-scale optimization $\left(P_{\mathrm{FS}}^{r t}\right)$.

TABLE IV

IPS STATE AND OPTIMIZATION PARAMETERs USED IN THE CASE STUdY

\begin{tabular}{|c|c|}
\hline Name & Value \\
\hline GT states $\left(x_{1, k}\right)$ & {$\left[m_{b, k}, T_{b, k}, \omega_{t c, k}\right]^{T}$} \\
\hline FC states $\left(x_{2, k}\right)$ & {$\left[W_{F R, i n, k}, T_{F R, k}, p_{H_{2}, a n, k}\right]^{T}$} \\
\hline Sample Times & $T_{f}=0.05 s, T_{s}=0.2 s$ \\
\hline Horizon Length & $N_{s}=200, N=800$ \\
\hline Tolerance Value & $\sigma=10^{-9}$ \\
\hline Terminal Cost & $h\left(x_{1, N}, x_{2, N}\right)=20\left(\frac{P_{d, N}-P_{1, N}-P_{2, N}}{P_{d, N}}\right)^{2}$ \\
\hline Instantaneous & $g\left(x_{1, k}, x_{2, k}, u_{1, k}, u_{2, k}\right)=$ \\
Cost function & $u_{1, k}^{2}+u_{2, k}^{2}+20\left(\frac{P_{d, k}-P_{1, k}-P_{2, k}}{P_{d, k}}\right)^{2}$ \\
\hline GT saturation, & {$\left[0.00385 m_{b, k} T_{b, k}-0.98\left(\omega_{t c, k}-19.483\right)\right.$,} \\
surge constraint & $0.137-u_{1, k}$, \\
$\Phi_{1}\left(x_{1, k}, u_{1, k}\right)$ & $\left.0.345-u_{1, k}\right]^{T} \leq \mathbf{0}_{3 \times 1}$ \\
\hline FC starvation & {$\left[0.04-\frac{p_{H_{2}, a n, k}}{p_{a n, k}}\right.$,} \\
saturation constraints & $0.022-u_{2, k}$, \\
$\Phi_{2}\left(x_{2, k}, u_{2, k}\right)$ & $\left.0.124-u_{2, k}\right]^{T} \leq \mathbf{0}_{3 \times 1}$ \\
\hline
\end{tabular}

TABLE V

REAL-TIME COMPUTATIONAL EFFORT REDUCTION USING TSS

\begin{tabular}{|c|c|c|c|}
\hline Method & $\begin{array}{c}\text { Real-time } \\
\text { Computation } \\
\text { Effort }(\mathrm{s})\end{array}$ & $\begin{array}{c}\text { Real-time } \\
\text { Cost }\end{array}$ & $\begin{array}{c}\text { Offline } \\
\text { Cost }\end{array}$ \\
\hline$F S$ & $\approx 1.6$ & 30.2 & 4.49 \\
\hline$L 1$ & $\approx 0.045$ & 4.6 & 4.6 \\
\hline
\end{tabular}

$P_{L 1}^{r t}, P_{\mathrm{FS}}^{r t}$ are associated with the optimal fuel trajectories using $L 1$ (blue dashed line denoted by) and FS (red dashed-dotted line) optimization. The power associated with the static feedforward control is optimized for steady state power tracking, where the presence of slow dynamics causes the mismatch between the response of the static optimal control and the actual demand. It must be noted that the control solutions of the $L 1$ optimization satisfies both hydrogen mole fraction and surge constraints, with the corresponding maximum values being 0 (active) and -0.45 , respectively.

\section{CONCLUSION}

In this paper, we proposed a multitime scale approach to solve the trajectory optimization problem associated with IPS PM in real-time. The use of this approach is beneficial in ensuring fast and efficient power tracking due to its realtime computational efficiency. We have implemented the proposed method on a real-time simulator and have demonstrated both improved real-time performance due to the control solutions being available within a sampling interval. This approach will provide the framework towards the dynamic reconfiguration of AES as the real-time feasibility of the PM problem is established.

\section{APPENDIX}

\section{A. Proof of Proposition 2.1}

We list the key ideas used in deriving Proposition 2.1. To determine the order of the SFM algorithm given in Section II, we first list the key tasks along with their flop count to compute control updates per iteration and the order of the algorithm is given by the dominant term to compute the control updates. For example, it can be shown the following.

- Performance index calculations: $N\left(p_{1}+p_{2}\right)$ flops, where $p_{1}, p_{2}$ is the flop count to compute $g\left(x_{k}, u_{k}\right)$ and $f\left(x_{k}, u_{k}\right)$, respectively. 
- Sensitivity functions calculations: $\mathrm{Np}_{4}+$ $(N(N+1)) /(2)\left(p_{5}+p_{6}\right)$ flops, where $p_{4}, p_{5}$ and $p_{6}$ is the flops to compute $\left(A_{k}, B_{k}, g_{x_{k}}, g_{u_{k}}\right)$, $\left(\partial x_{k+1}\right) /\left(\partial u_{i}\right)$ and the inner product $\left\langle G_{x}, Z_{i}\right\rangle$ given by $\sum_{k=i}^{N}(\partial g) /\left(\partial x_{k}\right)^{\prime}\left(\partial x_{k}\right) /\left(\partial u_{i}\right)$.

- Step size $\left(\delta^{*}\right)$ computation: $Q N\left(p_{1}+p_{2}\right)+(Q-1) p_{3}$ flops, where $p_{3}$ is the flop count for comparing floating point numbers and $Q$ is the number of points in the search space of $\delta$ at which we evaluate the cost, where we assume a brute force calculations.

- Updating all the control inputs requires $m(N-1)$ flops. The total FLOP count is given by $(N(N+1)) /(2)\left(p_{5}+p_{6}\right)+$ $N\left(p_{4}+(Q+1)\left(p_{1}+p_{2}\right)+(Q-1) p_{3}+m(N-1)\right.$. It can be seen that for $N \gg n, m$ the dominant term is the effort for SF generation, given as

$$
C_{S F}=\frac{N(N+1)}{2}\left(p_{5}+p_{6}\right)
$$

which is $\mathcal{O}\left(N^{2}\right)$.

\section{B. Proof of Corollary 2.1}

If we consider the band diagonal structure (See Remark 2.2), then the flop count for determining the $Z$ matrix and the inner product of $\left\langle G_{x}^{T}, Z_{i}\right\rangle$ is given by $\left(N_{B}\left(\left(N_{B}+1\right)\right) /(2)+N_{B}(N-\right.$ $\left.\left.N_{B}\right)\right)\left(p_{5}+p_{6}\right)$. Then for $N_{B} \gg 1$ the total effort for SFM algorithm can be given as

$$
C_{B D}=N_{B}\left(N-\frac{N_{B}}{2}\right)\left(p_{5}+p_{6}\right) .
$$

With a large length of horizon $\left(\left(N>N_{B}\right) \gg 1\right)$, we have $\left(C_{B D}\right) /\left(C_{S F}\right) \approx\left(2 N_{B}\right) /(N)\left(1-\left(N_{B}\right) /(2 N)\right)$, which is $\mathcal{O}\left(N_{B} / N\right)$.

\section{Proof of Proposition 2.2}

1) Part A: This follows from Proposition 2.1. Since $N_{s}=$ $\varepsilon N$, we have $C_{\text {slow }}=\mathcal{O}\left(N_{s}^{2}\right)=\varepsilon^{2} \mathcal{O}\left(N^{2}\right)$.

2) Part B: The flop count with the $L 2$ optimization that incudes level 1 slow time scale optimization as well as the fast time scale corrections is given by

$$
C_{T S}=C_{\text {slow }}+\beta\left(N-\frac{\beta}{2}\right)\left(p_{5}^{f}+p_{6}^{f}\right)
$$

where $\left(p_{5}^{f}, p_{6}^{f}\right)$ corresponds to the flop counts $\left(p_{5}, p_{6}\right)$ to compute $\left(\partial x_{k+1}\right) /\left(\partial u_{i}\right)$ and $\left\langle G_{x}^{T}, Z_{i}\right\rangle$ for the fast subsystems and $\beta \propto\left(\tau_{f}\right) /\left(T_{f}\right)$ is the number of band diagonal element of fast dynamics on the fast time scale. If $\alpha_{s}$ denotes the number of band diagonal elements of the slow dynamics on the slow time scale, by definition, we have $\beta=\varepsilon \gamma$ and $\alpha_{s}=\varepsilon \gamma$, where $\gamma$ denotes the number of band diagonal elements of the overall system dynamics on the fast time scale. Then we have

$$
\frac{C_{T S}}{C_{S F}}=\frac{C_{\text {slow }}}{C_{S F}}+\varepsilon \frac{N-\varepsilon \gamma / 2}{N-\gamma / 2}
$$

where $\left(C_{\text {slow }}\right) /\left(C_{S F}\right)=\mathcal{O}\left(\varepsilon^{2}\right)$ from Proposition 2.2. Under the assumption $\varepsilon \ll 1$, we have $\left(C_{T S}\right) /\left(C_{S F}\right)$ is $\mathcal{O}(\varepsilon)$ which proves the corollary.

\section{REFERENCES}

[1] T. J. McCoy, "Trends in ship electric propulsion," in Proc. IEEE Power Eng. Soc., 2002, pp. 343-346.

[2] N. Doerry and J. C. Davis, "Integrated power system for marine applications," Naval Eng. J., vol. 106, no. 3, pp. 77-90, 1994.

[3] Y. Xie, G. Seenumani, J. Sun, Y. Liu, and Z. Li, "A PC-cluster based real-time simulator for all-electric ship integrated power systems analysis and optimization," in Proc. IEEE Electric Ship Technol. Symp., 2007, pp. 396-401.

[4] V. Tsourapas, J. Sun, and A. Nickens, "Control oriented modeling and analysis of a hybrid solid oxide fuel cell and gas turbine (SOFC/GT) system," presented at the Fuel Cell Seminar, Honolulu, HI, 2006.

[5] J. Betts, "Survey of numerical methods for trajectory optimization," $J$. Guid., Control, Dyn., vol. 21, no. 2, pp. 193-207, 1998.

[6] D. P. Bertsekas, Dynamic Programming and Optimal Control. Nashua, NH: Athena Scientific, 1995.

[7] D. F. Opila, D. Aswani, R. McGee, J. A. Cook, and J. W. Grizzle, "Incorporating drivability metrics into optimal energy management strategies for hybrid vehicles," in Proc. Conf. Decision Control, 2008, pp. 4382-4389.

[8] P. Rodatz, G. Paganelli, A. Sciarretta, and L. Guzzella, "Optimal power management of an experimental fuel cell/supercapacitor-powered hybrid vehicle," Control Eng. Practice, vol. 13, no. 1, pp. 41-53, 2005.

[9] R. Fletcher, Practical Methods Of Optimization. Hoboken, NJ: Wiley, 1987.

[10] R. Ghaemi, J. Sun, and I. Kolmonovsky, "Model predictive control for constrained discrete time systems: An optimal perturbation analysis approach," in Proc. Amer. Control Conf., 2007, pp. 3757-3762.

[11] P. E. Gill, W. Murray, and M. A. Saunders, "SNOPT: An SQP algorithm for large-scale constrained optimization," in Proc. Soc. for Ind. Appl. Math. Rev., 2005, pp. 99-131.

[12] P. A. Ioannou and J. Sun, Robust Adaptive Control. Englewood Cliffs, NJ: Prentice-Hall, 1996.

[13] G. Seenumani, J. Sun, and H. Peng, "A numerically efficient iterative procedure for hybrid power system optimization using sensitivity functions," in Proc. Amer. Control Conf., 2007, pp. 4738-4743.

[14] M. S. Bazaraa, H. D. Sherali, and C. M. Shetty, Nonlinear Programming: Theory \& Algorithms. Hoboken, NJ: Wiley, 2005.

[15] G. Seenumani, "Real-time power management of hybrid power systems in all-electric ship applications," Ph.D. dissertation, Dept. Mech. Eng., Univ. Michigan, Ann Arbor, 2010.

[16] J. T. Pukrushpan, A. G. Stefanopoulou, and H. Peng, Control of Fuel Cell Power Systems. : Springer, 2004, ISBN 1852338164.

[17] G. G. Kulikov and H. A. Thompson, Dynamic Modelling of Gas Turbines. New York: Springer, 2004. 